\title{
PECULIARITIES OF RADIATION-INDUCED PROCESSES IN THE Cr-Ni-Mo AUSTENITIC STEELS STUDIED BY NEUTRON DIFFRACTION
}

\author{
V. I. Voronin, V. L. Arbuzov, V. I. Bobrovskii*, S. E. Danilov, K. A. Kozlov, N. V. Proskurnina, \\ V. V. Sagaradze
}

M.N. Miheev Institute of Metal Physics of Ural Branch of Russian Academy of Sciences, 18 S. Kovalevskoy st., Ekaterinburg, Russian Federation

*Corresponding author. E-mail: brodova@imp.uran.ru; address for correspondence: 620990, ul. S. Kovalevskoy, 18, Ekaterinburg, Russian Federation. Tel.: +7 343 3745244; fax: +7 3433745244

Understanding the mechanisms of radiation-induced phenomena in FCC-materials is of fundamental significance for the development of new austenitic reactor steels. An important role in these phenomena, along with the crystal structure and chemical composition of the matrix, belongs to doping elements and the microstructure of the material. In this paper, peculiarities of competing processes that proceed under fast neutron irradiation in $\mathrm{Cr}-\mathrm{Ni}-\mathrm{Mo}$ steels doped with $\mathrm{Ti}$ are studied by means of neutron diffraction. It is demonstrated that, on the one hand, new $\mathrm{Ni}_{3} \mathrm{Ti} \gamma^{\prime}$-phase particles are formed and, on the other hand, they dissolve to form interstitial Ti atoms. Besides, there is radiation-induced relaxation of microscopic stresses, which, in the case of large neutron fluences, overlaps with additional microstresses resulting from the accumulation of radiation-induced defects. The observed effects agree with the results we obtained for other austenitic steels.

Keywords: austenitic reactor steels, radiation damages, defects, radiation-induced processes, doping, neutron diffraction.

DOI: $10.17804 / 2410-9908.2015 .5 .080-089$

\section{References}

1. Zeman A., Kaiser R., Inozemtsev V., Beatty R.L. IAEA activities on coordinated research of structural materials for advanced reactor systems. Journal of Nuclear Materials, 2012, vol. 428, iss. 1-3. - P. 3-5. DOI: 10.1016/j.jnucmat.2012.06.024.

2. Voevodin V.N., Neklyudov I.M. Problems of radiation resistance of structural materials in nuclear power engineering. Visnik Kharkivskogo Universitetu. Ser. Fizika, 2006, no.746, iss.4, pp. 3-22. (In Russian).

3. Okita T., Wolfer W.G., Garner F.A., and Sekimura N. Effects of titanium additions to austenitic ternary alloys on microstructural evolution and void swelling. Philosophical Magazine, 2005, vol. 85, iss. 18, pp. 2033-2048. DOI: 10.1080/14786430412331331871.

4. David C., Panigrahi B.K., Balaji S., Balamurugan A.K., Nair K.G.M., Amarendra G., Sundar C.S., Baldev R. A study of the effect of titanium on the void swelling behavior of D9 steels by ion beam simulation. Journal of Nuclear Materials, 2008, vol. 383, iss. 1-2, pp.132-136. DOI: 10.1016/j.jnucmat.2008.08.049.

5. Sagaradze V.V., Goshchitskii B.N., Volkova E.G., Voronin V.I., Berger I.F., and Uvarov A.I. Evolution of the Microstructure and Microstresses in the 40Kh4G18F2 Steel upon Carbide Aging. Physics of Metals and Metallogragraphy, 2011, vol. 111, iss. 1, pp. 80-90. DOI: 10.1134/S0031918X1101011X.

6. Sagaradze V.V., Voronin V.I., Berger I.F., Volkova E.G., Goshchitskii B.N. Evolution of the microstructure and microdistortions in the austenitic $\mathrm{Cr}-\mathrm{Ni}-\mathrm{Ti}$ steel during aging. Physics of Metals and Metallogragraphy, 2011, vol. 12, iss. 5, pp. 517-525. DOI: 10.1134/S0031918X11050279. 
opent-access journal

7. Sagaradze V.V., Nalesnik V.M., Lapin S.S., Aliabev V.M. Precipitation hardening and radiation damageability of austenitic stainless steels. Journal of Nuclear Materials, 1993, vol. 202, iss. 1-2, pp.137-144. DOI: 10.1016/0022-3115(93)90036-X.

8. Krivoglaz M.A. X-ray and neutron diffraction in nonideal crystals. Berlin, Heidelberg, Springer Verlag Publ., 1996, 466 p.

9. Rodriguez-Carvajal J. Recent advances in magnetic structure determination by neutron powder diffraction. Physica B: Condensed Matter., 1993, vol.192, iss. 1-2, pp. 55-69. DOI: 10.1016/0921-4526(93)90108-I.

10. Ungar T., Borbely A. The effect of dislocation contrast on x-ray line broadening: a new approach to line profile analysis. Appl. Phys. Lett., 1996, vol. 69, iss. 21, pp.3173-3175. DOI: $10.1063 / 1.117951$.

11. Konobeevsky S.T. Deistvie oblucheniya na materialy [Effect of Irradiation on Materials]. M., Atomizdat Publ., 1967, 402 p. (In Russian).

12. Alab'yev V.M., Vologin V.G., Dubinin S.F., Lapin S.S., Parkhomenko V.D., Sagaradze V.V. Neutron diffraction and electron microscopic investigation of decomposition and radiationinduced ageing of Cr-Ni-Ti austenitic alloys. Physics of Metals and Metallography, 1990, vol. 70, iss. 2. pp. 131-137. 
Подана в журнал: 14.09 .2015

УДК 669.15-194.56:620.183.6:539.2:620.181

DOI: $10.17804 / 2410-9908.2015 .5 .080-089$

\title{
НЕЙТРОННО-ДИФРАКЦИОННЫЕ ИССЛЕДОВАНИЯ ОСОБЕННОСТЕЙ РАДИА- ЦИОННО-ИНДУЦИРОВАННЫХ ПРОЦЕССОВ В ХРОМ-НИКЕЛЬ- МОЛИБДЕНОВЫХ АУСТЕНИТНЫХ РЕАКТОРНЫХ СТАЛЯХ
}

\author{
В. И. Воронин, В. Л. Арбузов, В. И. Бобровский*, С. Е. Данилов, К. А. Козлов,
} Н. В. Проскурнина, В. В. Сагарадзе

\begin{abstract}
Федеральное государственное бюджетное учреждение науки Институт физики металлов имени М.Н. Михеева Уральского отделения Российской академии наук, ул. С. Ковалевской, 18, Екатеринбург, Российская Федерациия
\end{abstract}

*Ответственный автор. Электронная почта: bobrovskii@imp.uran.ru; адрес для переписки: 620990, ул. С. Ковалевской, 18, Екатеринбург, Российская Федерация. Телефон / факс: +7 (343) 374-00-03

Понимание механизмов радиационно-индуцированных явлений в ГЦК материалах имеет фундаментальное значение для разработки новых аустенитных реакторных сталей. В этих явлениях помимо кристаллической структуры и химического состава матрицы важнейшую роль играют легирующие примеси и микроструктурное состояние материалов. В работе средствами нейтронографии вскрыты особенности конкурентных процессов, протекающих при облучении быстрыми нейтронами в легированных титаном хром-никель-молибденовых сталях. Показано, что, с одной стороны, происходит образование новых частиц $\gamma^{\prime}-ф а з ы ~ \mathrm{Ni}_{3} \mathrm{Ti}$, а с другой - идет их растворение с образованием междоузельных атомов титана. Также протекают процессы радиационно-стимулированной релаксации микронапряжений, перекрывающиеся в случае больших нейтронных флюенсов возникновением дополнительных микронапряжений вследствие накопления радиационных дефектов. Наблюдавшиеся эффекты согласуются с результатами, полученными авторами для других аустенитных сталей.

Ключевые слова: аустенитные реакторные стали, радиационно-индуцированные процессы, легирование, нейтронная дифракция.

\section{1. Введение}

Разработка и производство конструкционных материалов для ядерных и термоядерных реакторов, удовлетворяющих жестким условиям эксплуатации в полях интенсивного реакторного облучения, представляет собой весьма актуальную и серьезную проблему $[1,2]$. Для оптимального выбора и определения направлений поисков перспективных материалов для атомной энергетики необходимо понимание особенностей формирования как их исходной микроструктуры в процессе технологических обработок, так и процессов и механизмов взаимодействия быстрых нейтронов с веществом. В настоящее время в качестве кандидатных материалов для быстрых реакторов исследуются различные типы сталей: ферритные, ферритно-мартенситные, аустенитные. В данной работе объектом наших исследований выбрана аустенитная реакторная сталь Х16Н15М3Т1. Это связано с сохраняющимся в мире высоким интересом к поиску способов улучшения свойств реакторных аустенитных сталей, поскольку эти материалы технологичны, обладают большим запасом пластичности и вязкости, хотя и имеют меньшее сопротивление вакансионному распуханию при высокодозном нейтронном облучении, чем стали с ОЦК решеткой.

Основными методами повышения радиационной стойкости реакторных сталей являются легирование их различными химическими элементами, а также термомеханические обработки, в результате чего в материале формируются системы ловушек, на которых проис- 
ходит рекомбинация точечных радиационных дефектов. Понимание строения таких систем и механизмов их функционирования дает ключ к созданию радиационно-стойких материалов.

Титан в настоящее время является одним из основных легирующих элементов аустенитных нержавеющих сталей. Ранее было установлено, что при внедрении Ті в аустенитную матрицу имеет место формирование наноразмерных карбидных выделений $\mathrm{TiC}$, сдерживающих радиационно-индуцированное вакансионное распухание [3, 4]. В процессе формирования этих выделений важную роль играют дислокации, и, как следствие, возникает необходимость тщательного подбора режимов предварительной термомеханической обработки материала и их контроля. Подобные процессы (но с карбидами ванадия) мы наблюдали ранее в высокоуглеродистой аустенитной стали 40Х4Г 18Ф2 [5] и в стали Н26X5Т3, где формировались наночастицы $\gamma^{\prime}$-фазы $\mathrm{Ni}_{3} \mathrm{Ti}$ [6], которые, как было показано в [7], также могут сдерживать радиационно-индуцированное распухание материалов.

Основными достоинствами нейтронографии как метода исследования наших объектов являются:

- глубина сканирования исследуемого материала намного превышает возможности других методов;

- одновременно измеряются средняя по объему деформация решетки и микронапряжения в пределах зерен;

- определяется кристаллографическая анизотропия деформаций;

- в случае многофазного материала определяются напряжения для каждой фазы;

- одновременно с внутренними напряжениями могут быть определены текстура материала, средние размеры когерентно рассеивающих областей и фазовый состав. В частности, нейтронографический анализ интерметаллида $\mathrm{Ni}_{3} \mathrm{Ti}$ в подобных сплавах более предпочтителен, чем электронно-микроскопический или рентгеновский, поскольку ядерные амплитуды когерентного рассеяния нейтронов для никеля и титана имеют разные знаки.

Эти достоинства проявились при исследовании образцов стали Х16Н15М3Т1. Конкретной целью этих экспериментов было отследить возможные изменения фазового состава материала, параметра решетки ГЦК-матрицы и возникающих в ней при обработках и облучении микроискажений.

\section{2. Материал и методика}

В работе исследуется аустенитная реакторная сталь Х16Н15М3Т1. Химический состав стали: $\mathrm{Cr}-16 \%$; $\mathrm{Ni}-15 \%$; Mo - 2,5\%; Ti - 1,02\%; $\mathrm{Si}-0,5 \%$; $\mathrm{Mn}-0,38 \%$; C - 0,03 \%; $\mathrm{Fe}$ - остальное.

После выплавки и гомогенизирующего высокотемпературного отжига $\left(1100{ }^{\circ} \mathrm{C}\right)$ образцы были закалены в воду, отпущены при $450{ }^{\circ} \mathrm{C}$, а затем состарены при температурах 600 и $700{ }^{\circ} \mathrm{C}$ в течение нескольких часов (от 1 до 12). Далее из обработанных таким образом заготовок были вырезаны цилиндрические образцы для нейтронографических исследований (диаметром 6 мм, длиной 40 мм). Облучение образцов флюенсом $1,2 \times 10^{20} \mathrm{H} / \mathrm{cm}^{2}$ при температуре $\sim 80{ }^{\circ} \mathrm{C}$ проводили в «мокрых» облучательных каналах исследовательского ядерного реактора ИВВ-2М. Величина плотности потока быстрых нейтронов энергией $\mathrm{E}_{\mathrm{n}} \geq 0,1 \mathrm{MэB}$ в «мокром» канале равна $2,23 \times 10^{14} \mathrm{H} / \mathrm{cm}^{2}$ сек $^{-1}$.

Нейтронно-дифракционные исследования выполнялись на дифрактометре высокого разрешения Д-7А $(\Delta \mathrm{d} / \mathrm{d}=0,2 \%)$ Нейтронного материаловедческого комплекса ИФМ УрО РАН на реакторе ИВВ-2M.

\section{3. Результаты и обсуждение}

На нейтронограммах всех образцов Х16Н15М3Т1 наблюдались только пики ГЦК твердого раствора. Для примера на рис.1 приведена нейтронограмма исходного закаленного образца.

Voronin V. I. et al. / Peculiarities of radiation-induced processes in the Cr-Ni-Mo austenitic steels studied by 


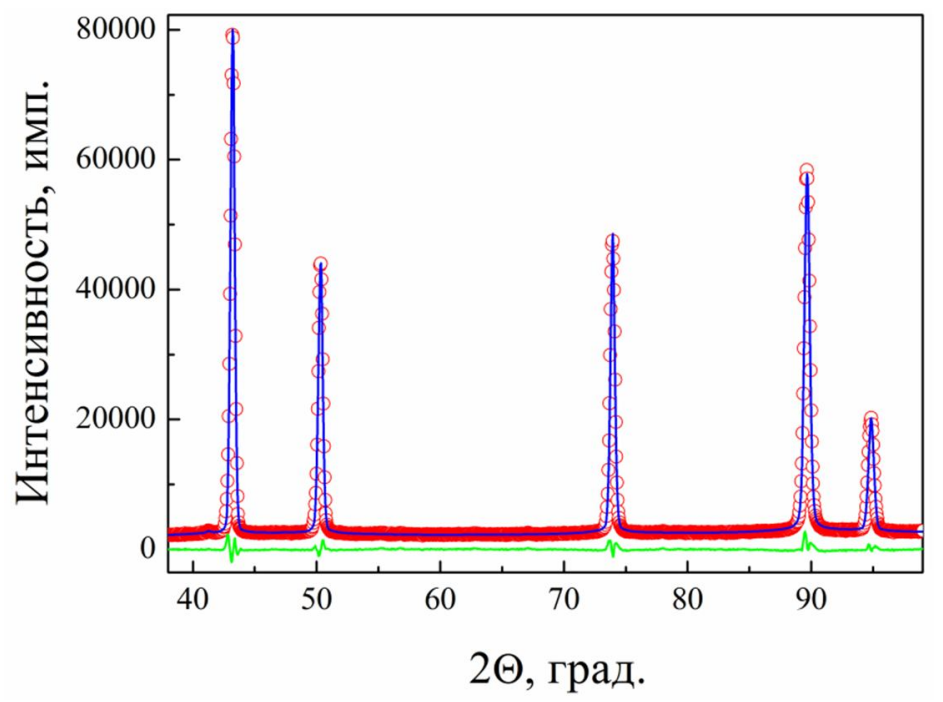

Рис. 1. Нейтронограмма образца стали Х16Н15М3Т1 при комнатной температуре: точки - экспериментальные данные; огибающая синяя линия - расчет; нижняя зеленая линия - разностная кривая между экспериментом и расчетом

Однако профильный анализ обнаружил небольшое уширение рефлексов. На рис.2 показан экспериментальный профиль рефлекса (311) и теоретический профиль исходного закаленного (принимаемого за эталон) образца. Видно, что экспериментальный рефлекс уширен и возникли «крылья», т.е. изменилась форма пика. Такие изменения, согласно теории рассеяния рентгеновских лучей и тепловых нейтронов [8], указывают на существование микронапряжений в объеме образца.

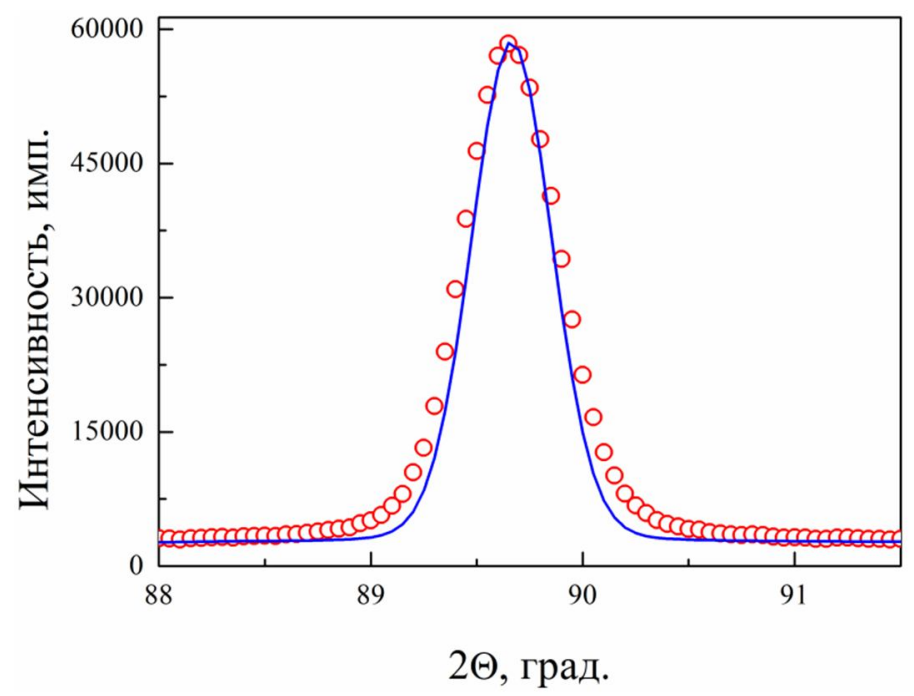

Рис. 2. Рефлекс (311) образца стали Х16Н15М3Т1: красные кружки - эксперимент; синяя линия - теоретический расчет эталона

Поэтому для обработки всего профиля нейтронограмм мы использовали компьютерную программу FulProf [9], которая позволяет рассчитать величину микронапряжений с использованием эталонной функции разрешения дифрактометра.

Анализ полученных экспериментальных нейтронограмм показал, что с увеличением времени старения при двух температурах $600{ }^{\circ} \mathrm{C}$ и $700{ }^{\circ} \mathrm{C}$ уменьшается параметр решетки, но различным образом. Основное изменение при температуре $600^{\circ} \mathrm{C}$ происходит в первый час, затем величина параметра практически не изменяется. При $700{ }^{\circ} \mathrm{C}$ максимальное падение па- 
open-aCcess journal

раметра происходит после 6-часового отжига, а при дальнейшем немного увеличивается. Зависимости этих величин приведены на рис. 3.

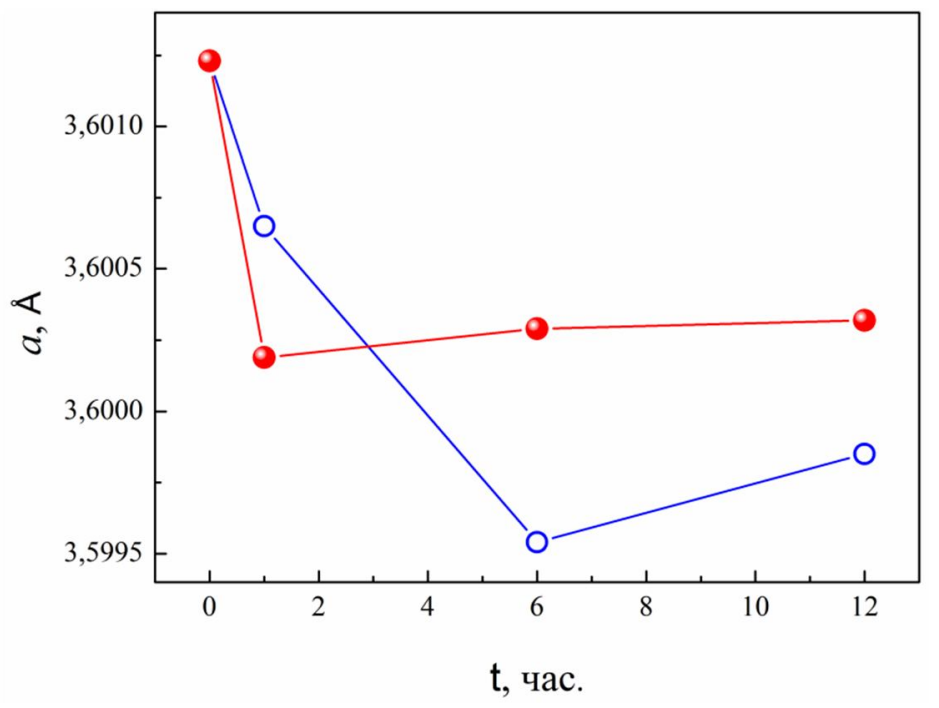

Рис. 3. Зависимость параметра ГЦК-решетки стали Х16N15М3T1 от времени старения при температурах $600^{\circ} \mathrm{C}$ (красный цвет) и $700^{\circ} \mathrm{C}$ (синий цвет)

Также было обнаружено, что с увеличением времени старения при обеих температурах изменяются величины микроискажений (рис. 4) и, соответственно, связанных с ними модулями упругости микронапряжений. Но, в отличие от параметра решетки, основное падение микроискажений после часового отжига наблюдается сразу в обоих образцах. При этом, однако, после 12-часового отжига при температуре $700{ }^{\circ} \mathrm{C}$ вновь наблюдается их рост. Отметим, что на рис. 4. представлены усредненные величины микроискажений, хотя более прецизионные исследования показывают, что наблюдается некоторая анизотропия в уширениях рефлексов. Анизотропия уширения может быть связана с возникновением дислокационной структуры в образцах [10], возникшей в процессе исходной термомеханической обработки материала. Подробный расчет плотности дислокаций предполагается в последующих исследованиях.

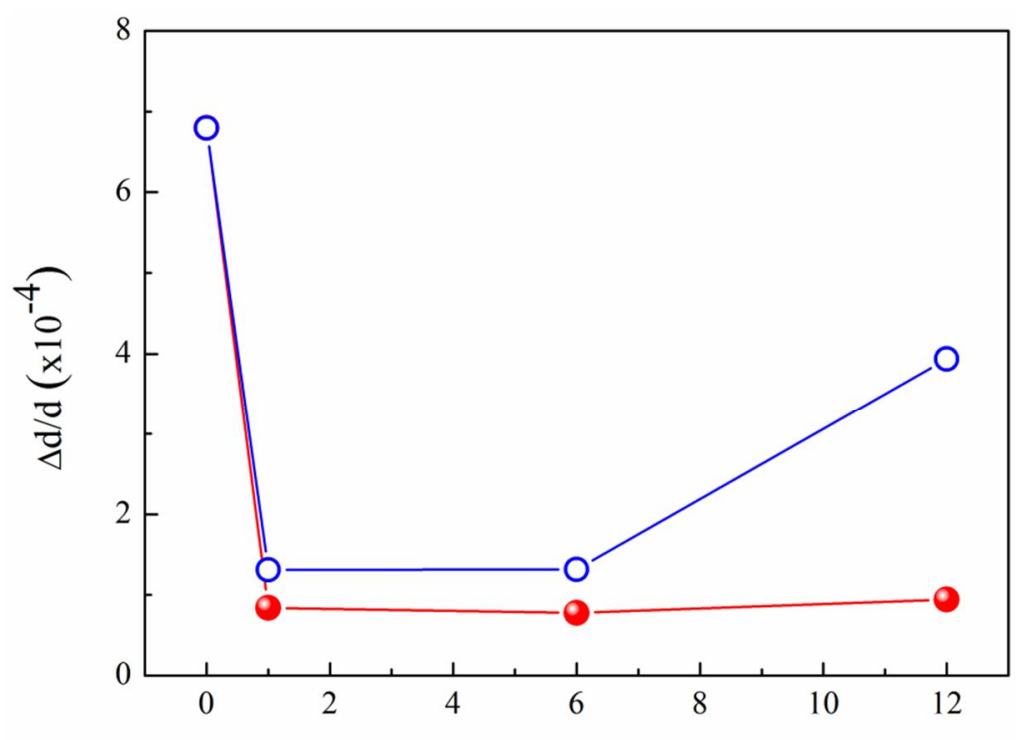

t, час.

Рис. 4. Зависимость микроискажений в стали X16N15M3T1 от времени старения при температурах $600^{\circ} \mathrm{C}$ (красный цвет) и $700^{\circ} \mathrm{C}$ (синий цвет) 
Как отмечено выше, семь образцов стали Х16Н15M3Т1 с различной подготовкой (после закалки от $1100{ }^{\circ} \mathrm{C}$ и отпуска при $450{ }^{\circ} \mathrm{C}$, после закалки и старения при $600{ }^{\circ} \mathrm{C}$ и $700{ }^{\circ} \mathrm{C}$ в течение 1; 6 и 12 ч) были облучены быстрыми нейтронами в канале реактора ИВВ-2М при температуре $\sim 80^{\circ} \mathrm{C}$ флюенсом $\Phi=1,2 \times 10^{20} \mathrm{~cm}^{-2}$.

На рис. 5 для примера приведены нейтронограммы закаленного (исходного) образца стали Х16Н15М3Т1 до и после облучения быстрыми нейтронами флюенсом $\Phi=12 \times 10^{19} \mathrm{~cm}^{-2}$. Из него видно, что после облучения быстрыми нейтронами наблюдается увеличение интенсивности фона, изменение углового положения рефлексов и понижение интенсивности рефлексов в больших углах рассеяния, которое характерно при увеличении амплитуды статических смещений атомов решетки из законных узлов. Аналогичные изменения наблюдаются и на других нейтронограммах облученных образцов.

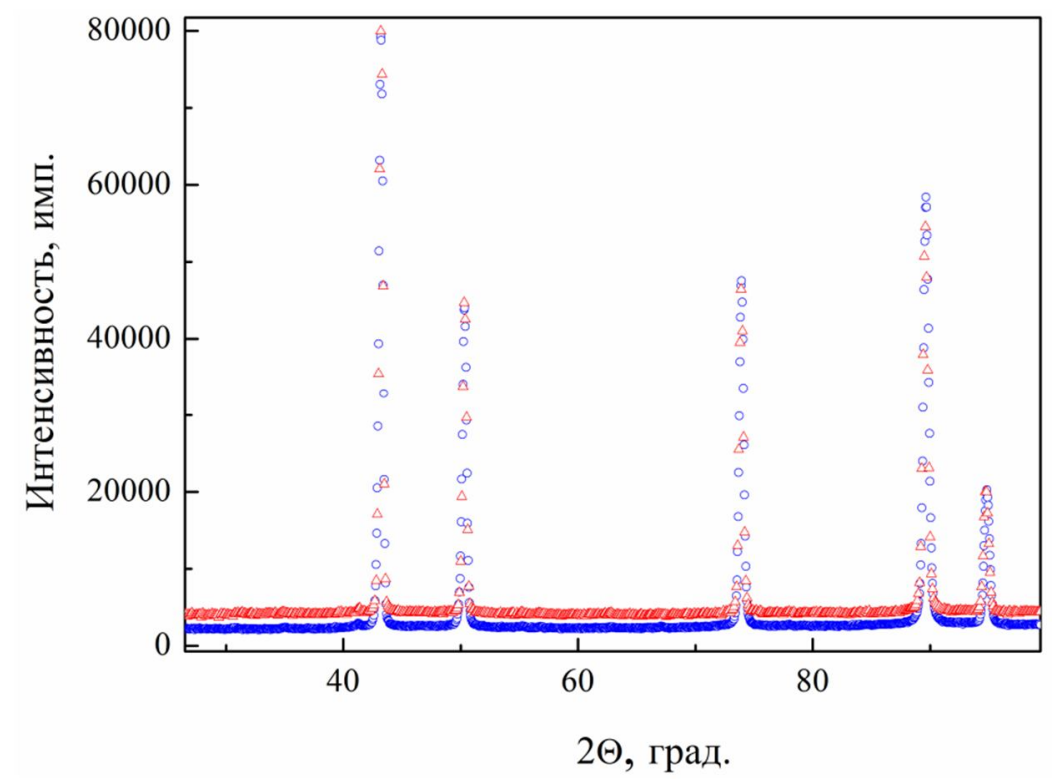

Рис. 5. Нейтронограммы закаленного образца стали Х16Н15М3Т1 до (синие кружки) и после облучения (красные треугольники)

Для получения прецизионной информации о структурных параметрах мы прибегли к профильному анализу полных нейтронограмм, результаты которого представлены в таблице.

Структурные параметры ГЦК-матрицы стали Х16Н15М3Т1 в зависимости от времени старения, температуры старения и облучения быстрыми нейтронами ( $a$ - параметр решетки; $\Delta \mathrm{d} / \mathrm{d}$ - микроискажения; В - тепловой фактор)

\begin{tabular}{|c|c|c|c|c|c|c|c|c|}
\hline Время, ч & \multicolumn{2}{|c|}{0} & \multicolumn{2}{|c|}{1} & \multicolumn{2}{|c|}{6} & \multicolumn{2}{|c|}{12} \\
\hline $\begin{array}{c}\text { Флюенс } \\
\left(10^{20} \mathrm{~cm}^{-2}\right)\end{array}$ & 0 & 1,2 & 0 & 1,2 & 0 & 1,2 & 0 & 1,2 \\
\hline \multicolumn{9}{|c|}{ Температура старения $600^{\circ} \mathrm{C}$} \\
\hline$a, \AA$ & 3,60121 & 3,59713 & 3,60019 & 3,59650 & 3,60029 & 3,59748 & 3,59985 & 3,59872 \\
\hline$\Delta \mathrm{d} / \mathrm{d}\left(10^{-4}\right)$ & 5,87 & 4,45 & 0,0 & 1,28 & 0,0 & 2,45 & 0,0 & 2,24 \\
\hline $\mathrm{B}, \AA^{2}$ & $0,39(1)$ & $0,58(2)$ & $0,35(1)$ & $0,58(2)$ & $0,29(1)$ & $0,56(2)$ & $0,31(1)$ & $0,66(2)$ \\
\hline$\Delta a, \AA$ & & 0,00408 & & 0,00369 & & 0,00281 & & 0,00113 \\
\hline \multicolumn{9}{|c|}{ Температура старения $700^{\circ} \mathrm{C}$} \\
\hline$a, \AA$ & 3,60121 & 3,59713 & 3,60064 & 3,59789 & 3,59955 & 3,59612 & 3,59985 & 3,59920 \\
\hline$\Delta \mathrm{d} / \mathrm{d}\left(10^{-4}\right)$ & 5,8746 & 4,45 & 0,3479 & 1,5523 & 0,3089 & 1,3445 & 0,0 & 0,8304 \\
\hline $\mathrm{B}, \AA^{2}$ & $0,39(1)$ & $0,58(2)$ & $0,33(1)$ & $1,00(3)$ & $0,32(1)$ & $0,35(3)$ & $0,33(1)$ & $1,04(2)$ \\
\hline$\Delta a, \AA$ & & 0,00408 & & 0,00275 & & 0,00353 & & 0,00065 \\
\hline
\end{tabular}

Voronin V. I. et al. / Peculiarities of radiation-induced processes in the Cr-Ni-Mo austenitic steels studied by 
Из таблицы видно, что, во-первых, параметр решетки уменьшается в целом как после временной выдержки при температурах, так и после облучения быстрыми нейтронами. Вовторых, величины тепловых параметров после температурных отжигов остаются примерно равными для всех образцов. Но в облученных образцах мы наблюдаем увеличение их значений. Этот факт указывает на возникновение в облученных образцах некоррелированных статических смещений атомов решетки из своих законных узлов. Наконец, мы видим уменьшение микронапряжений, которые имели значительную величину в исходном образце, после облучения, тогда как в образцах, термически обработанных, наоборот, наблюдается либо возникновение микронапряжений в объемах, либо возрастание их величин.

В исходном образце мы наблюдали, с одной стороны, однофазный твердый ГЦК раствор, а с другой стороны, анизотропное уширение рефлексов. По-видимому, это означает полное растворение легирующих атомов в решетке железа и образование дислокационной структуры, вызывающее это анизотропное уширение. При температурной обработке дислокации разрушаются, что приводит к сужению рефлексов и, соответственно, понижению или исчезновению внутренних микронапряжений. Небольшое уменьшение параметра решетки можно отнести к отжигу межузельных атомов. Уход титана из решетки маловероятен, т.к. по оценке выход титана (1\%) из узлов должен приводить к падению параметра решетки примерно на $0,005 \AA$, тогда как эксперимент дает максимум $0,0015 \AA$, т.е. величину в три раза меньше.

Иная картина наблюдается при облучении быстрыми нейтронами. Известно, что в процессе облучения материал подвергается комплексному воздействию: облучению быстрыми нейтронами различной энергии, а также температуры. В результате этого происходит изменение внутренних микронапряжений, могут выделяться или исчезать мелкодисперсные включения, а также увеличиваться или уменьшаться плотность дислокаций. Мы обнаружили, что при облучении исходного образца стали Х16H15М3Т1 флюенсом $1,2 \times 10^{20} \mathrm{H} / \mathrm{cm}^{2}$ параметр решетки уменьшился. Такое поведение в работе [11] объяснялось разной подвижностью межузельных атомов и вакансий. При невысоких температурах облучения (у нас $\left.80{ }^{\circ} \mathrm{C}\right)$ вакансии остаются неподвижными, тогда как подвижность межузельных атомов высока, и они в процессе облучения могут образовывать кластеры или выходить на границы зерен, поглощаться другими дефектами и т.п. Такие процессы приводят к уменьшению влияния межузельных атомов на расширение решетки, а отрицательный вклад вакансий, наоборот, увеличивается, понижая объем ячейки. В нашем случае, дополнительное уменьшение параметра решетки может быть связано также с процессами выпадения под облучением из твердого раствора наночастиц $(\mathrm{Fe} / \mathrm{Cr})_{3} \mathrm{Ti}$, эффектом, обнаруженным в сталях, обогащённых титаном $[6,12]$, и обусловленным тем, что атомы титана имеют больший размер, чем атомы железа. На нейтронограмме образца Х16Н15М3Т1, облученного высоким флюенсом $1.2 \times 10^{20} \mathrm{H} / \mathrm{cm}^{2}$, наблюдается небольшое уширение рефлексов. Угловая зависимость уширения рефлексов указывает на то, что оно вызвано возникновением микронапряжений в объеме образца, источником которых, согласно теории рассеяния рентгеновских лучей и тепловых нейтронов на несовершенствах в кристалле, развитой в работах М. Кривоглаза [8], как раз могут быть выпавшие наночастицы. Вероятно, с этими двумя механизмами и связано уменьшение параметров при облучении.

На нейтронограммах облученных образцов мы наблюдаем также увеличение изотропного фона по сравнению с состаренными образцами. Это увеличение фона, по-видимому, связано с возникновением радиационных дефектов. В нашем случае одним из типов радиационных дефектов являются некоррелированные статические смещения атомов из своих законных мест в ячейке. Согласно теории рассеяния нейтронов, проявлением таких дефектов является уменьшение интенсивности рефлексов в дальних углах, подобное связанному с тепловыми колебаниями атомов. И действительно, при обработке нейтронограмм облученных образцов лучшее согласие между расчетом и экспериментом достигается при увеличе-

Voronin V. I. et al. / Peculiarities of radiation-induced processes in the Cr-Ni-Mo austenitic steels studied by neutron diffraction. 
нии факторов Дебая - Валера (см таблицу). Его величина есть сумма вкладов тепловых колебаний и статических смещений атомов в решетке.

Таким образом, можно предположить два процесса, происходящих в облученных образцах. Первый - это уход большого по размерам атома внедрения (титана) из матрицы для образования $\gamma^{\prime}$-фазы $\left(\mathrm{Ni}_{3} \mathrm{Ti}\right)$, о чем свидетельствует уменьшение параметра решетки ГЦКматрицы. Второй - образование новых междоузельных атомов титана в процессе растворения интерметаллидов $\mathrm{Ni}_{3} \mathrm{Ti}$ при нейтронном облучении. Обратим внимание на высокую скорость падения параметра решетки с флюенсом в стали X16H15M3T1 с малым содержанием титана, хотя при старении этот параметр почти не изменяется (с точностью до 3 знака). Отсюда можно предположить, что все эти изменения параметра и его скорость обусловлены тем, что при облучении эффекту ухода атомов титана из решетки сопутствуют процессы формирования радиационных дефектов или «радиационно-стимулированные процессы разупорядочения», которых нет при термическом старении.

Такие фазовые превращения сказываются на структурном состоянии образцов, вызывая (увеличивая) или снимая (уменьшая) микронапряжения в объеме материала. В данном случае во всех образцах X16Н15M3T1 с начальными незначительными микроискажениями мы видим возрастание их величин с ростом флюенса во всем исследованном интервале, в отличие от систем 40Х4Г 18Ф2, Н26Х5Т3 [5,6] с достаточно большими исходными величинами внутренних напряжений, где при облучении небольшими флюенсами (до $5 \times 10^{19} \mathrm{~cm}^{-2}$ ) наблюдается частичное снятие напряжений и более явно проявляются два конкурирующих процесса. Один - это радиационно-стимулированная релаксация микронапряжений, которая происходит в результате диффузии атомов и аннигиляции дислокаций, и эта релаксация аналогична процессам диффузионного отжига при термическом воздействии. Этот процесс приводит к понижению микроискажений в материале. Второй, альтернативный, процесс - это создание радиационных дефектов в виде точечных дефектов, их скоплений и кластеров, а также в виде дислокационных петель, что вызывает рост микроискажений решетки. При наличии больших внутренних напряжений при малых флюенсах преобладает радиационностимулированная диффузия, уменьшающая величину микронапряжений, что мы и наблюдаем на эксперименте. При больших флюенсах преобладающим является процесс создания радиационных дефектов, приводящих к возникновению дополнительных микронапряжений в образцах. Отметим, что при всех процессах, как при старении, так и облучении быстрыми нейтронами, эволюция микронапряжений зависит от типа выделяющихся частиц и их концентрации.

\section{4. Заключение}

Полученные нами экспериментальные данные свидетельствуют о развитии под нейтронным облучением конкурентных процессов в системе ГЦК-матрица - микровключения интерметаллидной $\gamma^{\prime}$-фазы $\mathrm{Ni}_{3} \mathrm{Ti}$ в низколегированной титаном аустенитной стали X16H15M3T1. С одной стороны, происходит образование новых частиц $\gamma^{\prime}$-фазы, с другой идет их растворение с образованием междоузельных атомов титана. При этом протекает также радиационно-стимулированная релаксация микронапряжений, перекрывающаяся в случае больших нейтронных флюенсов возникновением вследствие накопления радиационных дефектов дополнительных микронапряжений. Существенную роль при этом играет предыстория образцов (в нашем случае искусственное старение). Эти явления, по-видимому, носят в аустенитных реакторных сталях довольно универсальный характер, поскольку согласуются с полученными ранее авторами результатами для других аустенитных сталей. Очевидный интерес и важное значение имеет получение систематической информации при легировании таких материалов другими химическими элементами, включая весьма возможные синергетические эффекты совместного легирования. 


\section{Благодарность}

Работа выполнена с использованием УНУ «НМК ИФМ» в рамках государственного задания ФАНО России (тема «Поток», № 01201463334) при частичной поддержке УрО РАН (проект № 15-17-2-3).

\section{Литература}

1. IAEA activities on coordinated research of structural materials for advanced reactor systems / A. Zeman, R. Kaiser, V. Inozemtsev, R. L. Beatty // Journal of Nuclear Materials. - 2012. Vol. 428, iss. 1-3. - P. 3-5. DOI: 10.1016/j.jnucmat.2012.06.024.

2. Воеводин В. Н., Неклюдов И. М. Проблемы радиационной стойкости конструкционных материалов ядерной энергетики // Вісник Харькивського университету. Сер. Физика. - 2006. - № 746, вып. 4. - С. 3-22.

3. Effects of titanium additions to austenitic ternary alloys on microstructural evolution and void swelling / T. Okita, W. G. Wolfer, F. A. Garner, and N. Sekimura // Philosophical Magazine. 2005. - Vol. 85, iss. 18. - P. 2033-2048. - DOI: 10.1080/14786430412331331871.

4. A study of the effect of titanium on the void swelling behavior of D9 steels by ion beam simulation / C. David, B. K. Panigrahi, S. Balaji, A. K. Balamurugan, K. G. M. Nair, G. Amarendra, C. S. Sundar, Raj Baldev // Journal of Nuclear Materials. - 2008. - Vol. 383, iss. 1-2. - P.132-136. - DOI: 10.1016/j.jnucmat.2008.08.049.

5. Evolution of the Microstructure and Microstresses in the 40Kh4G18F2 Steel upon Carbide Aging / V. V. Sagaradze, B. N. Goshchitskii, E. G. Volkova, V. I. Voronin, I. F. Berger, and A. I. Uvarov // Physics of Metals and Metallogragraphy. - 2011. - Vol. 111, iss. 1. - P. 80-90. DOI: $10.1134 / \mathrm{S} 0031918 X 1101011 \mathrm{X}$.

6. Evolution of the microstructure and microdistortions in the austenitic $\mathrm{Cr}-\mathrm{Ni}$ - $\mathrm{Ti}$ steel during aging / V. V. Sagaradze, V. I. Voronin, I. F. Berger, E. G. Volkova, B. N. Goshchitskii // Physics of Metals and Metallogragraphy. - 2011. - Vol. 12, iss. 5. - P. 517-525. DOI: $10.1134 / \mathrm{S} 0031918 \mathrm{X} 11050279$.

7. Precipitation hardening and radiation damageability of austenitic stainless steels / V. V. Sagaradze, V. M. Nalesnik, S. S. Lapin, V. M. Aliabev // Journal of Nuclear Materials. 1993. - Vol. 202, iss. 1-2. - P.137-144. - DOI: 10.1016/0022-3115(93)90036-X.

8. Krivoglaz M. A. X-ray and Neutron Diffraction in Nonideal Crystals. - Berlin, Heidelberg: Springer Verlag Publ., 1996. - 466 p.

9. Rodriguez-Carvajal J. Recent advances in magnetic structure determination by neutron powder diffraction // Physica B: Condensed Matter. - 1993. - Vol.192, iss. 1-2. - P. 55-69. DOI: 10.1016/0921-4526(93)90108-I.

10. Ungar T., Borbely A. The effect of dislocation contrast on x-ray line broadening: a new approach to line profile analysis // Appl. Phys. Lett. - 1996. - Vol. 69, iss. 21. - P. 3173-3175. DOI: $10.1063 / 1.117951$.

11. Конобеевский С. Т. Действие облучения на материалы. - М. : Атомиздат, 1967. - 402 с.

12. Neutron diffraction and electron microscopic investigation of decomposition and radiationinduced ageing of Cr-Ni-Ti austenitic alloys / V. M. Alab'yev, V. G. Vologin, S. F. Dubinin, S. S. Lapin, V. D. Parkhomenko, V. V. Sagaradze // Physics of Metals and Metallography. - 1990. Vol. 70, iss. 2. - P. 131-137. 